\title{
The Additive Effects of Type-2 Diabetes on Cognitive Function in Older Adults with Heart Failure
}

\author{
Michael L. Alosco, ${ }^{1}$ Mary Beth Spitznagel, ${ }^{1,2}$ Manfred van Dulmen, ${ }^{1}$ \\ Naftali Raz, ${ }^{3}$ Ronald Cohen, ${ }^{4}$ Lawrence H. Sweet, ${ }^{5}$ Lisa H. Colbert, ${ }^{6}$ \\ Richard Josephson, ${ }^{7,8,9}$ Joel Hughes, ${ }^{1,2}$ Jim Rosneck, ${ }^{2}$ and John Gunstad, 2 \\ ${ }^{1}$ Department of Psychology, Kent State University, Kent, OH 44242, USA \\ ${ }^{2}$ Department of Psychiatry, Summa Health System, Akron City Hospital, Akron, OH 44307, USA \\ ${ }^{3}$ Institute of Gerontology, Wayne State University, Detroit, MI 48202, USA \\ ${ }^{4}$ Department of Cardiology, Rhode Island Medical Center, Providence, RI 02903, USA \\ ${ }^{5}$ Department of Psychiatry and Human Behavior, Brown Medical School, Providence, RI 02903, USA \\ ${ }^{6}$ Department of Kinesiology, University of Wisconsin, Madison, WI 53706, USA \\ ${ }^{7}$ Department of Medicine, Case Medical Center, University Hospitals, Cleveland, OH 44106, USA \\ ${ }^{8}$ Harrington Heart \& Vascular Institute, Cleveland, OH 44106, USA \\ ${ }^{9}$ School of Medicine, Case Western Reserve University, Cleveland, OH 44106, USA
}

Correspondence should be addressed to Michael L. Alosco, malosco@kent.edu

Received 15 January 2012; Accepted 8 April 2012

Academic Editor: Jill Gelow

Copyright (c) 2012 Michael L. Alosco et al. This is an open access article distributed under the Creative Commons Attribution License, which permits unrestricted use, distribution, and reproduction in any medium, provided the original work is properly cited.

\begin{abstract}
Background. Medical comorbidity has been theorized to contribute to cognitive impairment in heart failure (HF) patients. Specifically, type-2 diabetes mellitus (T2DM), a common coexisting condition among HF patients, may be an independent predictor of cognitive impairment. Nonetheless, the relationships between T2DM and other risk factors for cognitive impairment among persons with HF are unclear. Methods. Persons with HF $(N=169,34.3 \%$ women, age $68.57 \pm 10.28$ years $)$ completed neuropsychological testing within a framework of an ongoing study. History of T2DM, along with other medical characteristics, was ascertained through a review of participants' medical charts and self-report. Results. Many participants (34.9\%) had a comorbid T2DM diagnosis. After adjustment for demographic and medical characteristics, HF patients with T2DM evidenced significantly greater impairments across multiple cognitive domains than HF patients without T2DM: $\lambda=.92, F(5,156)=2.82$, $P=.018$. Post hoc tests revealed significant associations between T2DM and attention $(P=.003)$, executive function $(P=.032)$, and motor functioning $(P=.008)$. Conclusion. The findings suggest additive contributions of T2DM and HF to impairments in attention, executive function, and motor function. Future work is needed to elucidate the mechanisms by which T2DM exacerbates cognitive impairment in HF.
\end{abstract}

\section{Introduction}

Heart failure (HF) affects nearly six million Americans and is associated with elevated rates of mortality $[1,2]$. HF is also the most common reason for rehospitalization $[3,4]$ and is linked to a number of negative outcomes, including poor quality of life [5] and reduced ability to perform instrumental activities of daily living $[6,7]$.

There is growing evidence that HF is also associated with poor neurocognitive outcomes. Patients with HF are at increased risk for Alzheimer's disease $[8,9]$, and cognitive impairment is found in as many as 75\% patients with HF [10]. Cognitive deficits are associated with functional impairment $[11,12]$ and increased risk of mortality in this population [13]. Many demographic and medical risk factors for cognitive impairment among older adults with HF have been identified, including older age [14], depression [15], hyperglycemia, history of stroke, elevated systolic blood pressure, metabolic abnormalities (i.e., low serum and anemia) [14], and high plasma brain natriuretic peptide [16], among many others. 
TABLE 1: Demographic and medical characteristics of older heart failure adults with and without type-2 diabetes mellitus (T2DM).

\begin{tabular}{lccc}
\hline Demographic characteristics & Heart failure w/o T2DM & Heart failure w/T2DM & Total sample \\
\hline$N$ & 110 & 59 & 169 \\
Age, mean (SD) & $68.85(11.18)$ & $68.05(8.42)$ & $68.57(10.28)$ \\
Gender (\% women) & 33.6 & 35.6 & 34.3 \\
Years of education, mean (SD) & $13.71(3.08)$ & $12.87(2.66)$ & $13.42(2.96)$ \\
Race (\% Caucasian) & 87.3 & 74.6 & 82.8 \\
\hline Medical characteristics & & & $60.99(24.06)$ \\
2MST, mean (SD) & $64.14(11.18)$ & $8.20(7.33)$ & $7.47(7.01)$ \\
BDI-II, mean (SD) & $7.08(6.83)$ & 5.1 & 8.9 \\
Stroke (\%) & 10.9 & 78.0 & 69.2 \\
Hypertension (\%) & 64.5 & 57.6 & 72.9 \\
MI (\%) & 54.5 & 63.6 & 66.9 \\
Elevated total cholesterol (\%) & 63.6 & \\
\hline
\end{tabular}

Note. 2MST: 2-minute step test; BDI II: beck depression inventory-II; MI: myocardial infarction.

Such findings highlight the important role of medical comorbidities in the etiology of cognitive impairment in persons with HF [14]. A condition frequently cooccurring in $\mathrm{HF}$ is type-2 diabetes mellitus (T2DM), with as many as $31 \%$ of patients with HF also having a comorbid T2DM diagnosis [17]. It is likely that T2DM could be an important contributor to cognitive dysfunction in HF, as patients with T2DM are at increased risk for cognitive impairment independent of HF $[18,19]$. One study showed that as many as $18 \%$ of community-residing diabetic older adults exhibit cognitive impairment and probable dementia [20]. In addition to specific impairments on neuropsychological tests of attention [21], executive function [22], and processing speed [23], older adults with diabetes also exhibit abnormalities on neuroimaging [23]. Moreover, a recent study demonstrated that when compared to HF patients without T2DM, HF patients with a coexisting T2DM diagnosis have poorer health, reduced quality of life, and shorter long-term survival [24].

Despite these findings, it remains unclear whether T2DM and HF have additive effects on cognitive performance. We examined this possibility in a sample of older adults with HF after adjusting for other important demographic variables and medical comorbidities. Secondary analyses also examined whether T2DM was associated with adverse psychosocial outcomes (i.e., quality of life and activities of daily living) among patients with HF. We hypothesized that HF patients with T2DM would exhibit significantly greater impairments across multiple cognitive domains and have poorer psychosocial outcomes than those without.

\section{Methods}

2.1. Participants. A sample of 175 consecutive persons with $\mathrm{HF}$ was selected from an ongoing NIH-funded study on cognitive function in HF. After data-screening procedures the sample size was reduced to 169 (see Section 2.4). Participants eliminated were not significantly different from the remaining sample in terms of gender $\left(\chi^{2}(1, N=175)=2.65\right.$, $P=.104)$, education $(t(173)=-.75, P=.453)$, depressive symptomatology (as assessed by the BDI-II) $(t(173)=1.60$, $P=.170)$, or history of diabetes $\left(\chi^{2}(1, N=175)=.58, P=\right.$ .448). However, those eliminated were significantly younger $(M=53.17, \mathrm{SD}=2.32$ versus $M=68.57, \mathrm{SD}=10.28)$ than the sample analyzed $(t(173)=-12.50, P=.000)$.

Participants were recruited from Summa Health System in Akron, $\mathrm{OH}$, USA and reflect the HF population receiving treatment at that facility. The inclusion criteria were age of 50-85 years, English as a primary language, and a diagnosis of New York Heart Association (NYHA) class II or III at the time of enrollment. NYHA history was confirmed by medical record review. NYHA is one of the most commonly used systems to classify symptoms of heart disease and is based on the following scale: (1) mild symptoms with no limitation of physical activity; (2) mild symptoms with slight limitation of physical activity, and ordinary physical activity may result in fatigue, heart palpitations, or shortness of breath; (3) noticeable limitations of physical activity and less than ordinary physical activity results in fatigue, heart palpitations, and shortness of breath; (4) severe limitations of physical activity with symptoms present at rest and increasing discomfort with physical activity [25].

Potential participants were excluded for history of significant neurological disorder (e.g., dementia), head injury with $>10$ minutes loss of consciousness, severe psychiatric disorder (e.g., schizophrenia and bipolar disorder), substance use, and renal failure. Participants, averaged $68.57 \pm 10.28$ years of age, were $34.3 \%$ female, and $82.8 \%$ Caucasian, $11.8 \%$ African American, 4.7\% Native American, and 0.6\% other. See Table 1 for demographic and medical information.

\subsection{Measures}

2.2.1. Neuropsychological Measures. All neuropsychological tests used in the current study have strong psychometric properties, including excellent reliability and validity. The domains and neuropsychological tests administered are as follows: (1) global cognitive function: Modified Mini Mental State Examination (3MS) [26]; (2) attention: Trail Making 
Test A [27] and Digit Symbol Coding [28]; (3) executive function: Trail Making Test B [29] and Letter Number Sequencing (LNS) [30]; (4) memory: The California Verbal Learning Test-II (CVLT-II) short delay free recall, long delay free recall and total hits [31]; (5) language: Boston Naming Test (BNT) [32] and Animal Fluency [33]; and (6) motor: Grooved Pegboard dominant and nondominant hand [29, $34,35]$.

2.2.2. Quality of Life. The Short Form-12 Quality of Life Measure (SF-12) [36] measures health-related quality of life. The two primary composite scores, Physical Composite Score (PCS; physical functioning, role-physical, bodily pain, and general health) and Mental Composite Scale (MCS; vitality, social functioning, role-emotional, and mental health) were used in the analyses.

2.2.3. Activities of Daily Living. The Lawton Brody Activities of Daily Living Scale were used to assess participants' reported instrumental and basic ADLs. This measure produces a total ADL score with an overall range between 0 and 28 , with higher scores indicative of functional independence [37].

2.2.4. Depressive Symptoms. The Beck Depression InventoryII [38] assessed depressive symptomatology in the current sample. The BDI-II is a commonly used measure with excellent psychometric properties in persons with medical conditions [39]. BDI-II scores range from 0 to 63 with higher scores indicative of increased symptomatology.

2.2.5. Demographic and Medical History. History of T2DM, along with other demographic and medical characteristics, was collected through a review of participants' medical charts and self-report. Specifically, a medical record review was conducted for all participants to corroborate self-report and to ascertain a physician diagnosis of T2DM. Refer to Table 1.

2.2.6. HF Severity. The 2-minute step test (2MST) is an assessment of cardiovascular endurance and was used to serve as an estimate of current heart failure severity [40]. The 2MST requires the patient to march in place for 2 minutes. The patient is asked to bring each knee up to a marked target set on the wall at the individual's own midpoint between the kneecap and crest of the iliac. The number of times that the right knee met the marked target was counted. Higher step count within the 2 minutes was reflective of greater cardiovascular fitness.

The 2MST has been suggested to be an alternative to the 6-minute walk test, which has been linked with functional work capacity, maximal oxygen uptake, and poor prognosis in patients with HF $[41,42]$. Recent work has also shown decreased performance on the 2MST to be associated with worse cognitive function in patients with HF [43]. In addition to these findings, the $2 \mathrm{MST}$ offers many practical advantages as it is brief, can be conducted within the confines of an examination room, and it is practical for patients with orthopedic devices or poor balance (i.e., patients are permitted to use the wall or a chair for support while performing the 2MST) [40,44].

2.3. Procedures. The local Institutional Review Board (IRB) approved the study procedures, and all participants provided written informed consent prior to study enrollment. Participants completed demographic, medical, and psychosocial self-report measures. A brief neuropsychological test battery was then administered to all heart failure participants to assess attention, executive function, memory, language, and motor functioning. Individuals then completed the 2MST under supervision.

2.4. Statistical Analyses. To facilitate clinical interpretation and to avoid undue influence of discrepancy in scales, all raw scores of the neuropsychological measures assessing cognitive function were transformed to $t$ scores (a distribution with a mean of 50, and a standard deviation of 10) using normative data correcting for age. Composite scores for attention, executive, memory, language, and motor functions were means of the $\mathrm{t}$ scores within each cognitive domain. Consistent with convention in many clinical settings, impairment in these domains for the current study was defined as a $t$ score of 1.5 standard deviations below the mean $(t<35)$. Screening of the data revealed outliers disrupting univariate normality on executive function, and motor domains, thus these cases were eliminated from analyses $(n=6)$.

To examine the additive effect of T2DM on cognitive impairment, a multivariate analysis of covariance (MANCOVA) was performed using attention, executive, memory, language, and motor functions as the dependent variables. Current medical history of T2DM served as the independent variable for each analysis. Demographic and medical variables including gender, education, depressive symptomatology (as assessed by the BDI-II), heart failure severity (as estimated by the 2MST), and history of stroke, hypertension, myocardial infarction, and elevated cholesterol were all entered as covariates. Age was not included as a covariate because the neuropsychological tests comprising the cognitive domains were corrected for age using normative data. A series of independent samples $t$-tests were then conducted to examine differences between older HF adults with and without T2DM on psychosocial measures assessing quality of life and activities of daily living.

\section{Results}

3.1. Demographic and Medical Differences between HF Patients with and without T2DM. Independent samples $t$ test and $\chi^{2}$ statistics were computed to identify differences between HF patients with and without T2DM on important demographic and medical variables. No significant betweengroup differences were found for age $(t(167)=-.483, P=$ $.629)$, gender $\left(\chi^{2}(1, N=169)=.07, P=.798\right)$, education $(t(167)=-1.76, P=.080)$, race $\left(\chi^{2}(3, N=169)=\right.$ $5.967, P=.113)$, or depressive symptomatology $(t(167)=$ $.99, P=.323)$. There were also no significant group differences on any of the medical comorbidities, including 
TABLE 2: Cognitive impairment in older heart failure adults with and without type 2 diabetes mellitus.

\begin{tabular}{|c|c|c|c|c|c|}
\hline $\begin{array}{l}\text { Cognitive } \\
\text { domain }\end{array}$ & $\begin{array}{c}\text { Total sample } \mathrm{T} \\
\text { score, mean }(\mathrm{SD}) \\
(N=169)\end{array}$ & $\begin{array}{c}\%<35 \text { T score } \\
\text { Total sample } \\
(N=169)\end{array}$ & $\begin{array}{c}\% \mathrm{HF} \text { w/T2DM T } \\
\text { score }<35 \\
(N=110)\end{array}$ & $\begin{array}{c}\% \text { HF w/o T2DM } \\
\text { T score }<35 \\
(N=59)\end{array}$ & $\chi^{2}(p)$ \\
\hline Attention & $48.40(9.62)$ & 7.7 & 11.9 & 5.5 & $2.22(.14)$ \\
\hline $\begin{array}{l}\text { Executive } \\
\text { Function }\end{array}$ & $47.36(11.32)$ & 11.2 & 15.3 & 9.1 & $1.46(.23)$ \\
\hline Memory & $45.89(9.56)$ & 10.7 & 10.2 & 10.9 & $.02(.88)$ \\
\hline Language & $51.66(11.15)$ & 8.3 & 11.9 & 6.4 & $1.53(.22)$ \\
\hline Motor & $36.91(14.18)$ & 34.3 & 49.2 & 26.4 & $8.85(<.01)$ \\
\hline
\end{tabular}

Abbreviations: HF w/T2DM: heart failure with type-2 diabetes mellitus; HF w/o T2DM: heart failure without type-2 diabetes mellitus.

history of hypertension $\left(\chi^{2}(1, N=169)=3.25, P=.072\right)$, myocardial infarction $\left(\chi^{2}(1, N=169)=.15, P=.701\right)$, or total elevated cholesterol $\left(\chi^{2}(1, N=169)=1.48, P=.224\right)$. However, when compared to HF patients without T2DM, HF patients with T2DM performed significantly worse on the 2MST (i.e., evidenced poorer cardiovascular fitness) $(t(167)=-2.35, P=.020)$. Additionally, although there were no statistically significant between group differences on history of stroke at the $P<.05$ level $\left(\chi^{2}(1, N=169)=1.61\right.$, $P=.204)$, HF patients without T2DM were nearly twice as likely to have a positive history of stroke than HF patients with T2DM. See Table 1 for details. However, it is important to note that all individuals were carefully screened for the presence of any neurological disorder likely to influence cognitive test performance. As such, patients identified with history of "stroke" were more accurately diagnosed with terms such as "cerebrovascular disease" and "white matter disease" rather than the stereotypic features of a cortical stroke (i.e., loss of language, hemiparesis, etc.).

3.2. Cognitive Impairment. T2DM was common in the current sample of HF patients, with $34.9 \%$ having a positive medical history of T2DM. Cognitive impairment was also common, as the sample mean 3MS score was $92.79 \pm 5.36$. Specifically, $24.3 \%$ of the participants had a $3 \mathrm{MS}$ score below 90, and 39.0\% had a 3MS score between 90 and 95 and $36.7 \%$ of the sample had a 3MS score between 95 and 100 . HF patients with T2DM performed worse on the $3 \mathrm{MS}$ than HF patients without T2DM $(t(167)=-2.02, P=.045)$. HF patients with T2DM had an average $3 \mathrm{MS}$ score of $91.66 \pm 5.37$ $(N=59)$, whereas HF patients without T2DM had an average $3 \mathrm{MS}$ of $93.39 \pm 5.28(N=110)$.

By a T-score cutoff of 35, many HF patients could be classified as impaired on tests of attention, executive function, memory, language, and motor functioning. Impairment in motor functioning $\left(\chi^{2}(1, N=169)=8.85, P=.003\right)$ was more common in HF patients with T2DM than those without (see Table 2).

3.3. T2DM and Cognitive Function in Older Adults with HF. After adjusting for gender, education, BDI-II, 2MST, and history of stroke, hypertension, myocardial infarction, and elevated cholesterol MANCOVA revealed a significant main effect of T2DM diagnosis across multiple cognitive domains:
$\Lambda=.92, F(5,156)=2.82, P=.018$. Post hoc tests showed HF patients with T2DM had poorer performance on tests of attention $(F(8,160)=9.29, P=.003)$, executive function $(F(8,160)=4.69, P=.032)$, and motor functioning $(F(8$, $160)=7.30, P=.008)$. No such pattern emerged for memory $(P=.635)$ or language $(P=.599)$. See Table 3 for a full summary of group differences in cognitive performance.

\subsection{T2DM and Performance on Specific Neuropsychological} Tests. Follow-up MANCOVA analyses adjusting for gender, education, BDI-II, 2MST, and history of stroke, hypertension, myocardial infarction, and elevated cholesterol were performed to clarify the relationship between T2DM and performance on neuropsychological tests assessing attention, executive function, and motor functioning. Bonferronicorrected posttests significance level, set at $P=.017$, revealed that HF patients with T2DM performed significantly worse than those without T2DM on Digit Symbol Coding $(F(9$, $159)=11.29, P=.001)$, Trail Making Test $\mathrm{B}(F(9,159)=$ $6.63, P=.011)$, and Grooved Pegboard with dominant $(F(9$, $159)=6.34, P=.013)$, and nondominant $(F(9,159)=6.68$, $P=.011)$ hands.

3.5. T2DM and Psychosocial Outcomes in Older Adults with $H F$. A series of independent samples $t$-tests revealed group differences in quality of life and ADL function as assessed by the SF-12 PCS $(t(167)=-2.59, P=.011)$ and total ADL $(t(99.96)=-2.01, P=.048)$ scores. HF patients with T2DM reported significantly poorer physical quality of life and reduced ability to perform ADLs than HF patients without T2DM. No such pattern emerged on the SF-12 MCS $(t(167)=-.52, P=.605)$. See Table 4 for details.

\section{Discussion}

Consistent with past work, cognitive impairment was common in current sample of older adults with HF. Medical comorbidities among patients with HF have been linked with increased risk of cognitive impairment in this population, including hypertension and hyperglycemia [14, 45]. The current study extends these findings by showing that older adults with $\mathrm{HF}$ and T2DM have greater impairments in cognitive function than those with HF alone. Such findings suggest 
TABLE 3: $T$-score means and standard deviations of cognitive test performance for heart failure patients with and without type-2 diabetes mellitus.

\begin{tabular}{|c|c|c|c|c|c|c|}
\hline \multicolumn{7}{|c|}{ Cognitive domains } \\
\hline & Attention & $\begin{array}{l}\text { Executive } \\
\text { function }\end{array}$ & Memory & Language & & Motor \\
\hline $\begin{array}{l}\mathrm{HF} \text { w/T2DM } \\
(N=110)\end{array}$ & 44.57 (9.64) & $43.81(13.24)$ & $45.31(9.15)$ & $50.03(13.22)$ & & 31.87 (14.89) \\
\hline $\begin{array}{l}\mathrm{HF} \text { w/o } \\
\text { T2DM } \\
(N=59)\end{array}$ & $50.45(8.99)$ & $49.26(9.67)$ & $46.20(9.80)$ & $52.53(9.82)$ & & $39.61(13.07)$ \\
\hline$F$ & $9.29^{* *}$ & $4.69^{*}$ & .23 & .28 & & $7.30^{* *}$ \\
\hline \multicolumn{7}{|c|}{ Performance on neuropsychological tests of attention, executive function, and motor } \\
\hline & TMTA & Digit & TMTB & LNS & PegsD & PegsND \\
\hline $\begin{array}{l}\mathrm{HF} \text { w/T2DM } \\
(N=110)\end{array}$ & $45.60(13.45)$ & $43.54(8.23)$ & $38.17(20.31)$ & 49.45 (8.39) & $29.51(18.54)$ & $34.23(12.51)$ \\
\hline $\begin{array}{l}\mathrm{HF} \text { w/o } \\
\text { T2DM } \\
(N=59)\end{array}$ & $51.17(10.70)$ & $49.72(9.19)$ & $47.16(13.49)$ & $51.38(9.40)$ & $38.56(15.76)$ & $40.67(11.83)$ \\
\hline$F$ & $4.53^{*}$ & $11.29^{\Psi}$ & $6.63^{\Psi}$ & .28 & $6.34^{\Psi}$ & $6.68^{\Psi}$ \\
\hline
\end{tabular}

Note. ${ }^{*}=P<.05 ; * *=P<.01 ;{ }^{\Psi}=P<.017$.

Abbreviations: HF w/T2DM: heart failure with type-2 diabetes mellitus; HF w/o T2DM: heat failure without type-2 diabetes mellitus; TMTA: trail making test A; digit: digit symbol coding; TMTBL: trail making test B; LNS: letter number sequencing; PegsD: grooved pegboard dominant hand; PegND: grooved pegboard nondominant hand.

TABLE 4: Means and standard deviations of psychosocial outcomes among older heart failure adults with and without type 2 diabetes mellitus.

\begin{tabular}{lccc}
\hline & SF-12 PCS & SF-12 MCS & Total ADL \\
\hline HF w/T2DM & $42.10(8.78)$ & $51.72(9.64)$ & $24.54(3.60)$ \\
$(N=110)$ & & & $25.54(9.97)$ \\
HF w/o T2DM & $45.49(7.74)$ & & $25.64(2.93)$ \\
$(N=59)$ & & $-.52(167)$ & $-2.13(99.96)^{*}$ \\
$t(d f)$ & $-2.59(167)^{*}$ & &
\end{tabular}

Note. ${ }^{*}=P<.05$.

Abbreviations: HF w/T2DM: heart failure with type-2 diabetes mellitus; HF w/o T2DM: heart failure without type-2 Diabetes mellitus; SF-12 PCS: SF-12 physical composite score; SF-12 MCS: SF-12 mental composite scale; Total ADL: total score on activities of daily living.

that T2DM may be an additional risk factor for cognitive impairment in this population.

The current study suggests that HF patients with T2DM have additive impairments in cognitive functioning, including attention, executive function and motor functioning. Patients with diagnosis of either HF or T2DM exhibit high rates of cognitive impairment in these domains $[10,17]$. Moreover, elevated fasting glucose in clinically normal adults is associated with reduced performance in executive functions [46]. Past work on the etiology of cognitive impairment in persons with HF has identified reduced cerebral perfusion as a result of left ventricular dysfunction [47] and a series of comorbid conditions (e.g., hyperglycemia and stroke history) [14] as important factors. The current findings suggest that T2DM is also an important contributor to cognitive impairment in HF. Such findings are perhaps not surprising given the growing evidence for adverse neurocognitive outcomes in persons with T2DM. For example, poor glycemic control and resulting cerebral microvascular and macrovascular damage (including reduced endothelial functioning) among patients with T2DM have been shown to be associated with cognitive impairment $[20,21,48-$ 50]. Interestingly, $\mathrm{HF}$ is associated with increased risk of Alzheimer's disease $(\mathrm{AD})[8,9]$, and the high insulin levels often observed in patients with T2DM have also been correlated with greater levels of amyloid-beta protein-an early indicator of $\mathrm{AD}$ pathogenesis [51-53]. Future work is needed to clarify the mechanisms by which HF and T2DM may interact.

Follow-up analyses showed that HF patients with T2DM demonstrated greater impairments on a task of psychomotor speed and speeded tasks of attention and executive function. Indeed, past work has shown T2DM patients (independent of $\mathrm{HF}$ ) to be associated with reduced performance on speeded neuropsychological measures and poorer psychomotor efficiency [54, 55]. Consistent with these findings, patients with T2DM often exhibit a cognitive pattern consisting of decreased mental speed and flexibility [56]. Increased cognitive slowing is an early indicator of cognitive impairment in dementia [57]; thus, future studies should examine whether HF patients with T2DM are at elevated risk for dementia. 
The current study found no additive effect of T2DM on memory in older adults with HF. While HF has been linked to impairments in memory, past work suggests that these deficits may be a result of an interaction between older age and low ejection fraction [58]. Similarly, past work has linked T2DM to memory impairment, with poor test performance being closely associated with longer T2DM duration and disease progression $[53,59]$. The exact reason for the absence of an additive effect of T2DM on memory in the current sample is unclear. One possible explanation is the absence of direct measures of glycemic control and insulin resistance in the current study. Recent studies suggest that many HF patients exhibit impaired insulin resistance [60] and that this process may occur independent of body composition [61]. As a result, it is possible that participants in the HF-only group of the current study actually exhibit some degree of insulin resistance, and these processes have recently been associated with poorer cognitive function [62]. Future work is needed to clarify this and other possible explanations.

In addition to poorer cognitive function, the current findings also indicate that HF patients with T2DM are at greater risk for reduced functional abilities and poorer quality of life. Such findings are consistent with past work demonstrating HF patients with T2DM to have reduced health-related quality of life [24]. Cognitive impairment may have a profound effect in the treatment management of T2DM in HF patients (i.e., poorer adherence to medication; dietary and physical activity recommendations). For instance, recent work found that cognitive impairment is associated with reduced functional independence in older adults with HF [6], and other work shows greater cognitive impairment among older adults with T2DM is related with poorer disease care management and adherence [63]. Additionally, the current study's findings that HF patients with T2DM reported that a significantly worse physical quality of life, as opposed to mental quality of life, than HF patients without T2DM has significant implications. For example, previous animal studies have shown physical activity to improve insulin resistance and attenuate neuroautonomic dysregulation among rats with cardiovascular disease and $\mathrm{HF}$ $[64,65]$. Future work should investigate whether physical activity in humans also produces similar metabolic changes and the possibility that such changes would reduce the adverse effects of T2DM on cognitive function in $\mathrm{HF}$ patients. Similarly, future studies should also explore the possibility that additive deficits in cognitive function due to multiple comorbidities may be an important cause for adverse psychosocial outcomes in persons with both HF and other common medical conditions in older adults.

Several limitations of the current study merit brief review. First, the present study consisted of cross-sectional data and prospective studies are needed to examine the trajectory of cognitive decline to clarify the relationship of T2DM and cognitive impairment in HF. Additionally, future studies are needed to identify the mechanisms of the adverse effects of T2DM on cognitive function among older adults with HF. Past work suggests cognitive impairment in patients with T2DM to be a result of the negative effects of hyperglycemia and glycation end products on the vascular system, including reduced cerebral blood flow and poorer endothelial functioning [21]. In addition, hyperinsulinemia in T2DM patients has also been linked with cerebral amyloid and tau metabolism [66]. Future studies should examine whether HF patients with T2DM are at greater risk of cognitive impairment through these and other mechanisms.

Finally, the current study examined $\mathrm{HF}$ as a broad disease entity, and specific type of HF (i.e., diastolic or systolic) was not examined. While previous research has shown systolic and diastolic HF to both be associated with worse cognitive function [67], the nature by which these $\mathrm{HF}$ etiologies interact with T2DM to produce cognitive dysfunction may be distinct. Future work should examine the additive effects of T2DM across HF types. Similarly, the current study examined HF severity using the 2MST, which has been linked with cognitive function in HF persons [43] and also has several practical advantages over commonly used walk tests. However, examining the interaction between T2DM and HF severity using measures such as VO2 max, cardiac output, and/or ejection fraction would provide clearer insight into the effects of T2DM on cardiac functioning. Additionally, future studies should also investigate the contribution of insulin resistance to cognitive function within $\mathrm{HF}$ patients using more sophisticated measures (e.g., HbA1C, oral glucose tolerance testing) and to also investigate disease duration. Lastly, the relatively small sample size in the current study did not permit analyses to control for key variables such as medication status and larger samples of HF patients with T2DM that are needed to further elucidate the independent additive effects of T2DM on cognitive function in HF.

In brief summary, the current study demonstrates that HF patients with T2DM have greater cognitive impairments than HF patients without T2DM. Future work is needed to elucidate the mechanisms by which T2DM exacerbates cognitive impairment in older adults with HF.

\section{References}

[1] V. L. Roger, S. A. Weston, M. M. Redfield et al., "Trends in heart failure incidence and survival in a community-based population," The Journal of the American Medical Association, vol. 292, no. 3, pp. 344-350, 2004.

[2] W. H. Barker, J. P. Mullooly, and W. Getchell, "Changing incidence and survival for heart failure in a well-defined older population, 1970-1974 and 1990-1994," Circulation, vol. 113, no. 6, pp. 799-805, 2006.

[3] S. F. Jencks, M. V. Williams, and E. A. Coleman, "Rehospitalizations among patients in the medicare fee-for-service program," The New England Journal of Medicine, vol. 360, no. 14, pp. 1418-1428, 2009.

[4] Lloyd-Jones et al., "Heart disease and stroke statistics-2010 update: a report from the American Heart Association," Circulation, vol. 121, no. 12, Article ID e260, 2010.

[5] S. J. Bennett, N. B. Oldridge, G. J. Eckert et al., "Comparison of quality of life measures in heart failure," Nursing Research, vol. 52, no. 4, pp. 207-216, 2003.

[6] M. L. Alosco, M. B. Spitznagel, R. Cohen et al., "Cognitive impairment is independently associated with reduced instrumental activities of daily living in persons with heart failure," The Journal of Cardiovascular Nursing, vol. 27, no. 1, pp. 4450, 2012. 
[7] Y. Seo, B. L. Roberts, I. Piña, and M. Dolansky, "Predictors of motor tasks essential for daily activities among persons with heart failure," Journal of Cardiac Failure, vol. 14, no. 4, pp. 296302, 2008.

[8] F. Rengo, D. Acanfora, L. Trojano et al., "Congestive heart failure in the elderly," Archives of Gerontology and Geriatrics, vol. 23, no. 3, pp. 201-223, 1996.

[9] C. Qiu, W. Xu, B. Winblad, and L. Fratiglioni, "Vascular risk profiles for dementia and Alzheimer's disease in very old people: a population-based longitudinal study," Journal of Alzheimer's Disease, vol. 20, no. 1, pp. 293-300, 2010.

[10] R. L. C. Vogels, P. Scheltens, J. M. Schroeder-Tanka, and H. C. Weinstein, "Cognitive impairment in heart failure: a systematic review of the literature," European Journal of Heart Failure, vol. 9, no. 5, pp. 440-449, 2007.

[11] G. Zuccalà, G. Onder, C. Pedone et al., "Cognitive dysfunction as a major determinant of disability in patients with heart failure: results from a multicentre survey," Journal of Neurology Neurosurgery and Psychiatry, vol. 70, no. 1, pp. 109-112, 2001.

[12] J. D. Putzke, M. A. Williams, F. J. Daniel, R. C. Bourge, and T. J. Boll, "Activities of daily living among heart transplant candidates: neuropsychological and cardiac function predictors," Journal of Heart and Lung Transplantation, vol. 19, no. 10, pp. 995-1006, 2000.

[13] G. Zuccalà, C. Pedone, M. Cesari et al., "The effects of cognitive impairment on mortality among hospitalized patients with heart failure," American Journal of Medicine, vol. 115, no. 2, pp. 97-103, 2003.

[14] G. Zuccalà, E. Marzetti, M. Cesari et al., "Correlates of cognitive impairment among patients with heart failure: results of a multicenter survey," American Journal of Medicine, vol. 118, no. 5, pp. 496-502, 2005.

[15] P. M. Pullicino, V. G. Wadley, L. A. McClure et al., "Factors contributing to global cognitive impairment in heart failure: results from a population-based cohort," Journal of Cardiac Failure, vol. 14, no. 4, pp. 290-295, 2008.

[16] F. Mauro, G. L. Rosso, M. Peano et al., "Correlation between cognitive impairment and prognostic parameters in patients with congestive heart failure," Archives of Medical Research, vol. 38, no. 2, pp. 234-239, 2007.

[17] J. B. Braunstein, G. F. Anderson, G. Gerstenblith et al., "Noncardiac comorbidity increases preventable hospitalizations and mortality among medicare beneficiaries with chronic heart failure," Journal of the American College of Cardiology, vol. 42, no. 7, pp. 1226-1233, 2003.

[18] R. Stewart and D. Liolitsa, "Type 2 diabetes mellitus, cognitive impairment and dementia," Diabetic Medicine, vol. 16, no. 2, pp. 93-112, 1999.

[19] A. Ott, R. P. Stolk, F. van Harskamp, H. A. P. Pols, A. Hofman, and M. M. B. Breteler, "Diabetes mellitus and the risk of dementia: the Rotterdam study," Neurology, vol. 53, no. 9, pp. 1937-1942, 1999.

[20] D. G. Bruce, G. P. Casey, V. Grange et al., "Cognitive impairment, physical disability and depressive symptoms in older diabetic patients: the Fremantle Cognition in Diabetes Study," Diabetes Research and Clinical Practice, vol. 61, no. 1, pp. 5967, 2003.

[21] S. Maggi, F. Limongi, M. Noale et al., "Diabetes as a risk factor for cognitive decline in older patients," Dementia and Geriatric Cognitive Disorders, vol. 27, no. 1, pp. 24-33, 2009.

[22] A. L. Fischer, C. M. de Frias, S. E. Yeung, and R. A. Dixon, "Short-term longitudinal trends in cognitive performance in older adults with type 2 diabetes," Journal of Clinical and
Experimental Neuropsychology, vol. 31, no. 7, pp. 809-822, 2009.

[23] A. L. Christman, T. D. Vannorsdall, G. D. Pearlson, F. HillBriggs, and D. J. Schretlen, "Cranial volume, mild cognitive deficits, and functional limitations associated with diabetes in a community sample," Archives of Clinical Neuropsychology, vol. 25, no. 1, pp. 49-59, 2010.

[24] B. Fujita, A. Lauten, B. Goebel et al., "Impact of diabetes mellitus on quality of life in patients with congestive heart failure," Quality of Life Research. In press.

[25] American Heart Association, "Classes of Heart Failure," 2012, http://www.heart.org/HEARTORG/Conditions/HeartFailure/ AboutHeartFailure/Classes-of-Heart-Failure_UCM_306328_ Article.jsp\#.T2od2pi4JSU.

[26] E. L. Teng and H. C. Chui, "The Modified Mini-Mental State (MMS) examination," Journal of Clinical Psychiatry, vol. 48, no. 8, pp. 314-318, 1987.

[27] O. Spreen and E. Strauss, A Compendium of Neuropsychological Tests, Oxford University Press, New York, NY, USA, 1991.

[28] A. Smith, "Clinical psychological practice and principals of neuropsychological assessment," in Handbook of Clinical Psychology: Theory, Research, and Practice, C. Walker, Ed., Dorsey Press, Homewood, Ill, USA, 1983.

[29] S. S. Dikmen, R. K. Heaton, I. Grant, and N. R. Temkin, "Testretest reliability and practice effects of expanded halsteadreitan neuropsychological test battery," Journal of the International Neuropsychological Society, vol. 5, no. 4, pp. 346-356, 1999.

[30] D. Wechsler, Manual for the Wechsler Adult Intelligence Scale, The Psychological Corporation, San Antonio, Tex, USA, 3rd edition, 1997.

[31] D. Delis, J. Kramer, E. Kaplan, and B. Ober, California Verbal Learning Test, Psychological Corporation, San Antonio, Tex, USA, 2nd edition, 2000.

[32] K. A. Hawkins, W. H. Sledge, J. F. Orleans, D. M. Quinlan, J. Rakfeldt, and R. E. Hoffman, "Normative implications of the relationship between reading vocabulary and Boston Naming Test performance," Archives of Clinical Neuropsychology, vol. 8, no. 6, pp. 525-537, 1993.

[33] J. C. Morris, A. Heyman, R. C. Mohs et al., "The consortium to establish a registry for Alzheimer's disease (CERAD). Part I. Clinical and neuropsychological assessment of Alzheimer's disease," Neurology, vol. 39, no. 9, pp. 1159-1165, 1989.

[34] H. Klove, "Clinical neuropsychology," in The Medical Clinics of North America, F. M. Forster, Ed., Saunders, New York, NY, USA, 1963.

[35] R. M. Ruff and S. B. Parker, "Gender- and age-specific changes in motor speed and eye-hand coordination in adults: normative values for the finger tapping and grooved pegboard tests," Perceptual and Motor Skills, vol. 76, no. 3, pp. 1219-1230, 1993.

[36] J. E. Ware, M. Kosinski, and S. D. Keller, "A 12-item short-form health survey: construction of scales and preliminary tests of reliability and validity," Medical Care, vol. 34, no. 3, pp. 220233, 1996.

[37] M. P. Lawton and E. M. Brody, "Assessment of older people: self-maintaining and instrumental activities of daily living," Gerontologist, vol. 9, no. 3, pp. 179-186, 1969.

[38] A. T. Beck, R. A. Steer, and G. K. Brown, Beck Depression Inventory, Psychological Corporation, San Antonio, Tex, USA, 2nd edition, 1996.

[39] R. C. Arnau, M. W. Meagher, M. P. Norris, and R. Bramson, "Psychometric evaluation of the beck depression inventory-II with primary care medical patients," Health Psychology, vol. 20, no. 2, pp. 112-119, 2001. 
[40] C. J. Jones and R. E. Rikli, "Measuring functional fitness of older adults," The Journal on Active Aging, pp. 24-30, 2002.

[41] P. A. Nixon, M. L. Joswiak, and F. J. Fricker, "A six-minute walk test for assessing exercise tolerance in severely ill children," The Journal of Pediatrics, vol. 129, no. 3, pp. 362-366, 1996.

[42] V. Bittner, D. H. Weiner, S. Yusuf et al., "Prediction of mortality and morbidity with a 6-minute walk test in patients with left ventricular dysfunction," The Journal of the American Medical Association, vol. 270, no. 14, pp. 1702-1707, 1993.

[43] M. L. Alosco, M. B. Spitznagel, N. Raz et al., "The 2-minute step test is independently associated with cognitive function in older adults with heart failure," Aging and Clinical Experimental Research. In press.

[44] A. Rózańska-Kirschke, P. Kocur, M. Wilk, and P. Dylewicz, "Fullerton fitness test as fitness index for the elderly," Rehabilitacja Medyczna, vol. 10, no. 2, pp. 9-16, 2006.

[45] L. Trojano, R. A. Incalzi, D. Acanfora, C. Picone, P. Mecocci, and F. Rengo, "Cognitive impairment: a key feature of congestive heart failure in the elderly," Journal of Neurology, vol. 250, no. 12, pp. 1456-1463, 2003.

[46] C. L. Dahle, B. S. Jacobs, and N. Raz, "Aging, vascular risk, and cognition: blood glucose, pulse pressure, and cognitive performance in healthy adults," Psychology and Aging, vol. 24, no. 1, pp. 154-162, 2009.

[47] P. M. Pullicino and J. Hart, "Cognitive impairment in congestive heart failure? Embolism vs hypoperfusion," Neurology, vol. 57, no. 11, pp. 1945-1946, 2001.

[48] M. J. Legato, A. Gelzer, R. Goland et al., "Gender-specific care of the patient with diabetes: review and recommendations," Gender Medicine, vol. 3, no. 2, pp. 131-158, 2006.

[49] A. P. Cannatà, M. Alberoni, M. Franceschi, and C. Mariani, "Frontal impairment in subcortical ischemic vascular dementia in comparison to Alzheimer's disease," Dementia and Geriatric Cognitive Disorders, vol. 13, no. 2, pp. 101-111, 2002.

[50] L. C. Perlmuter, M. K. Hakami, and C. Hodgson-Harrington, "Decreased cognitive function in aging non-insulin-dependent diabetic patients," American Journal of Medicine, vol. 77, no. 6, pp. 1043-1048, 1984.

[51] W. Farris, S. Mansourian, Y. Chang et al., "Insulin-degrading enzyme regulates the levels of insulin, amyloid $\beta$-protein, and the $\beta$-amyloid precursor protein intracellular domain in vivo," Proceedings of the National Academy of Sciences of the United States of America, vol. 100, no. 7, pp. 4162-4167, 2003.

[52] G. S. Watson, E. R. Peskind, S. Asthana et al., "Insulin increases CSF A $\beta 42$ levels in normal older adults," Neurology, vol. 60, no. 12, pp. 1899-1903, 2003.

[53] J. Hardy and D. J. Selkoe, "The amyloid hypothesis of Alzheimer's disease: progress and problems on the road to therapeutics," Science, vol. 297, no. 5580, pp. 353-356, 2002.

[54] S. E. Yeung, A. L. Fischer, and R. A. Dixon, "Exploring effects of type 2 diabetes on cognitive functioning in older adults," Neuropsychology, vol. 23, no. 1, pp. 1-9, 2009.

[55] C. M. Ryan, T. M. Williams, T. J. Orchard, and D. N. Finegold, "Psychomotor slowing is associated with distal symmetrical polyneuropathy in adults with diabetes mellitus," Diabetes, vol. 41, no. 1, pp. 107-113, 1992.

[56] A. M. A. Brands, G. J. Biessels, E. H. F. de Haan, L. J. Kappelle, and R. P. C. Kessels, "The effects of type 1 diabetes on cognitive performance: a meta-analysis," Diabetes Care, vol. 28, no. 3, pp. 726-735, 2005.

[57] R. A. Dixon, D. D. Garrett, T. L. Lentz, S. W. S. MacDonald, E. Strauss, and D. F. Hultsch, "Neurocognitive markers of cognitive impairment: exploring the roles of speed and inconsistency," Neuropsychology, vol. 21, no. 3, pp. 381-399, 2007.
[58] J. R. Festa, X. Jia, K. Cheung et al., “Association of low ejection fraction with impaired verbal memory in older patients with heart failure," Archives of Neurology, vol. 68, no. 8, pp. 10211026, 2011.

[59] O. I. Okereke, J. H. Kang, N. R. Cook et al., "Type 2 diabetes mellitus and cognitive decline in two large cohorts of community-dwelling older adults," Journal of the American Geriatrics Society, vol. 56, no. 6, pp. 1028-1036, 2008.

[60] C. Lazzeri, S. Valente, and P. Attanà, "Acute hyperglycemia and insulin resistance in acute heart failure syndromes without previously known diabetes," Internal and Emergency Medicine. In press.

[61] W. Doehner, D. Gathercole, M. Cicoira et al., "Reduced glucose transporter GLUT4 in skeletal muscle predicts insulin resistance in non-diabetic chronic heart failure patients independently of body composition," International Journal of Cardiology, vol. 138, no. 1, pp. 19-24, 2010.

[62] Z. S. Tan, A. S. Beiser, C. S. Fox et al., "Association of metabolic dysregulation with volumetric brain magnetic resonance imaging and cognitive markers of subclinical brain aging in middle-aged adults: the framingham offspring study," Diabetes Care, vol. 34, no. 8, pp. 1766-1770, 2011.

[63] D. G. Feil, A. Pearman, T. Victor et al., "The role of cognitive impairment and caregiver support in diabetes management of older outpatients," International Journal of Psychiatry in Medicine, vol. 39, no. 2, pp. 199-214, 2009.

[64] E. Cipolletta, A. Campanile, G. Santulli et al., "The G protein coupled receptor kinase 2 plays an essential role in betaadrenergic receptor-induced insulin resistance," Cardiovascular Research, vol. 84, no. 3, pp. 407-415, 2009.

[65] G. Rengo, D. Leosco, C. Zincarelli et al., "Adrenal GRK2 lowering is an underlying mechanism for the beneficial sympathetic effects of exercise training in heart failure," American Journal of Physiology, vol. 298, no. 6, pp. H2032-H2038, 2010.

[66] S. Craft and G. S. Watson, "Insulin and neurodegenerative disease: shared and specific mechanisms," The Lancet Neurology, vol. 3, no. 3, pp. 169-178, 2004.

[67] K. van den Hurk, Y. D. Reijmer, E. van den Berg et al., "Heart failure and cognitive function in the general population: the Hoorn study," European Journal of Heart Failure, vol. 13, no. 12, pp. 1362-1369, 2011. 


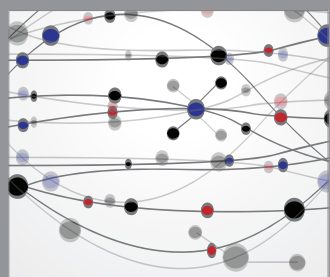

The Scientific World Journal
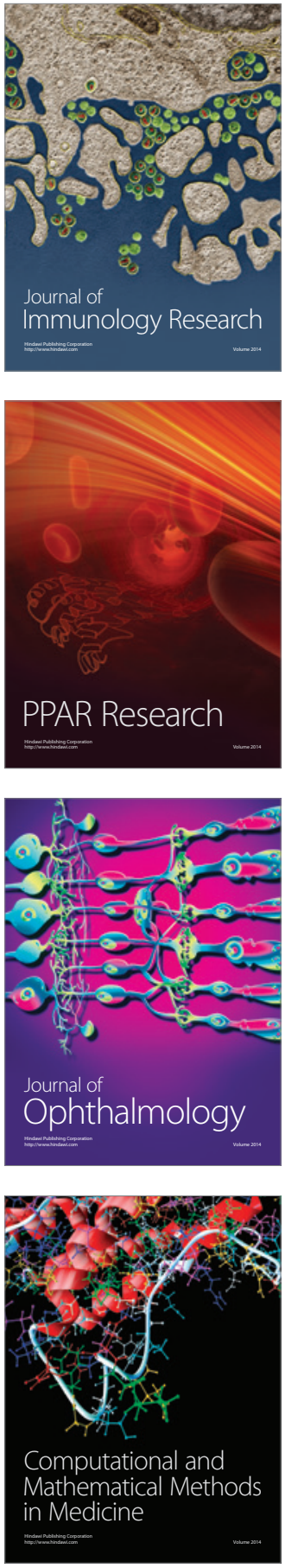

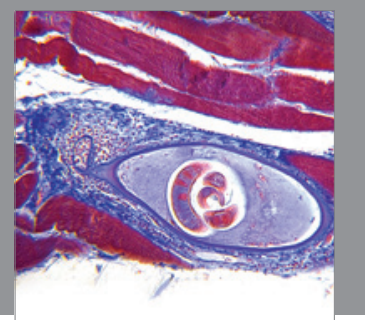

Gastroenterology

Research and Practice
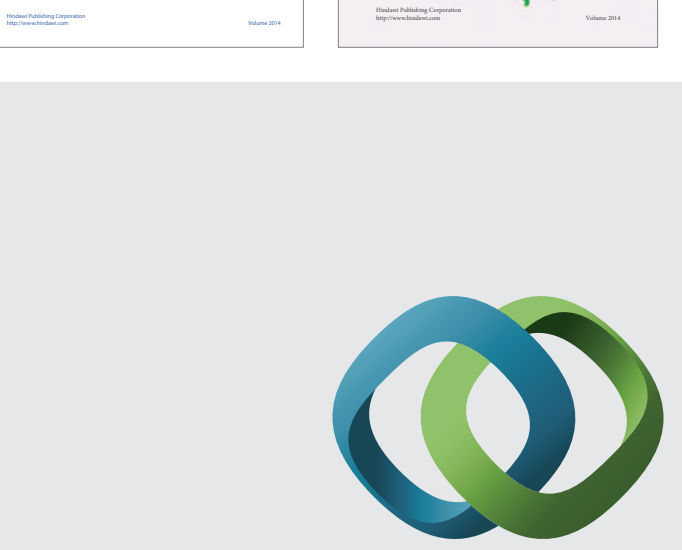

\section{Hindawi}

Submit your manuscripts at

http://www.hindawi.com
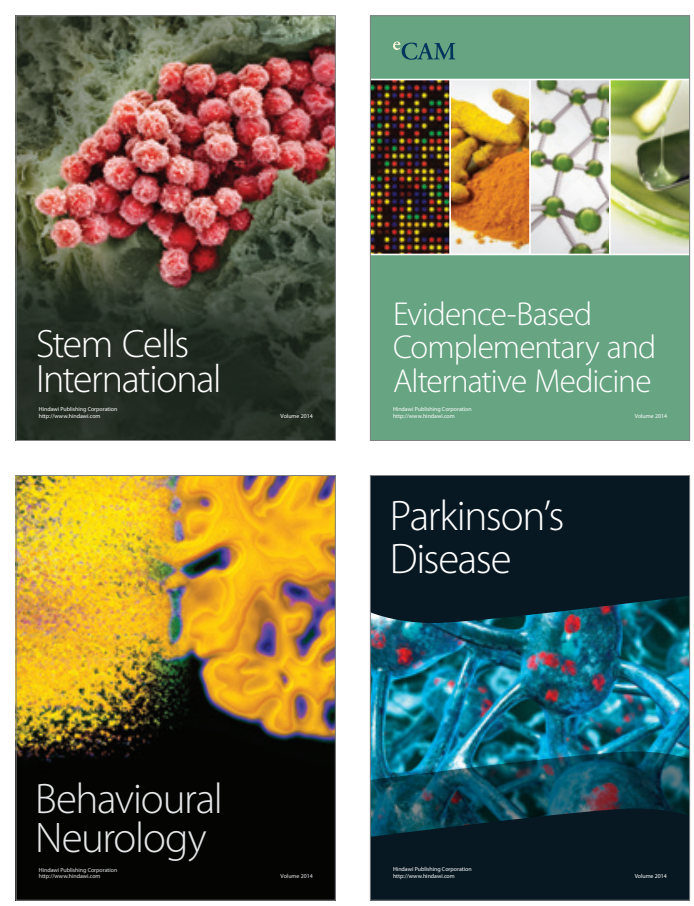

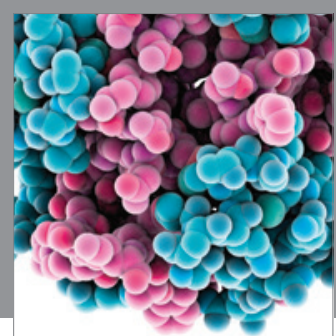

Journal of
Diabetes Research

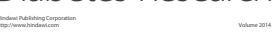

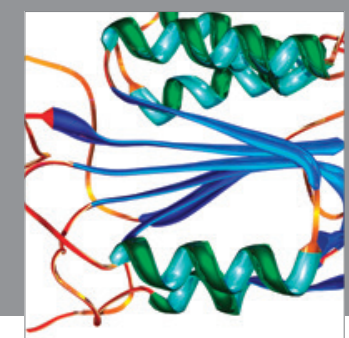

Disease Markers
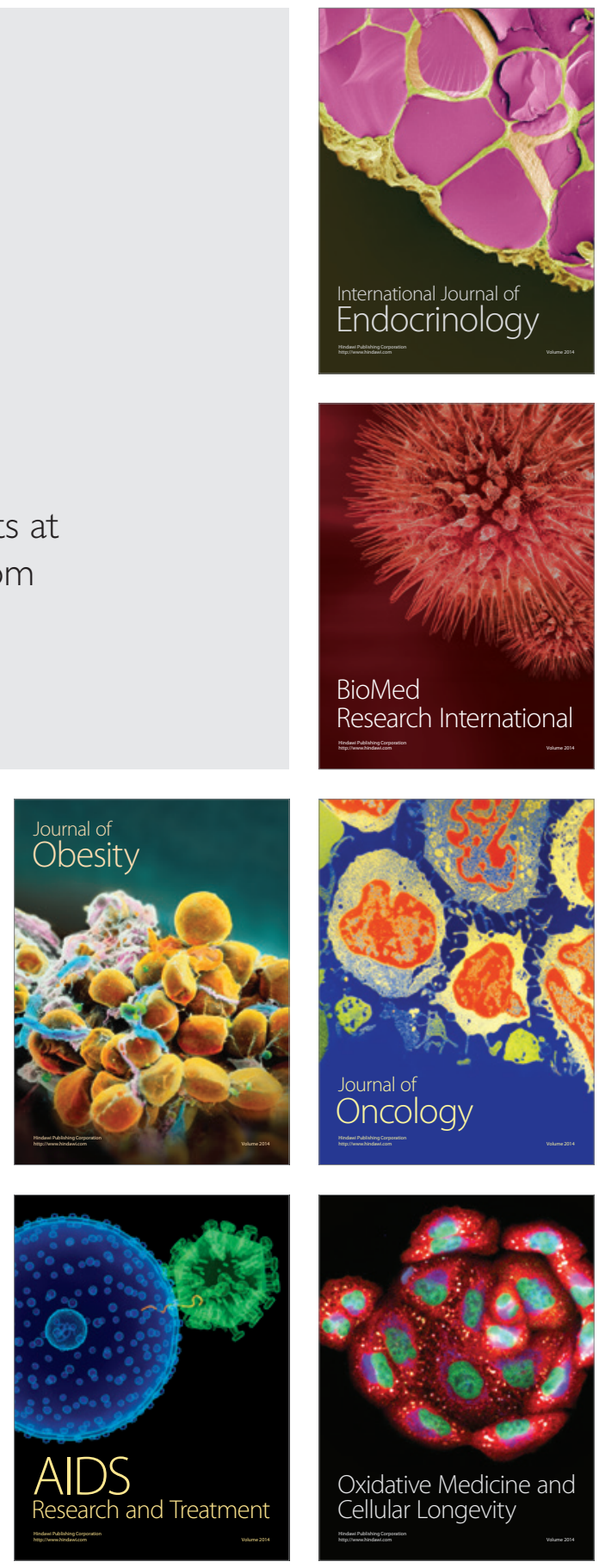